\title{
Methods and hints to linearise the resistance values vs. bending angle relationship of bend sensors
}

\author{
Giovanni Saggio \\ Dept. of Electronic Eng., Univ. of Rome "Tor Vergata" \\ via del Politecnico 1, Rome, Italy \\ saggio@uniroma2.it
}

\begin{abstract}
The correct measure of static and dynamic postures of patients is a fundamental element for dispensing correct rehabilitation procedures. Nowadays there are different sensors and transducers useful to reach the aim of measuring human postures, even in a non uncomfortable way, during normal activities of everyday life. Among all these sensors we selected the flex ones stand their cheapness an good performances in terms of reliability and stability of electrical signal they provide. It is possible to measure flex-extension of human joints simply laying the flex sensors on wrist, knee, elbow, ankle, etc. But a drawback is paid for these sensors, because of a non linear function of their electrical resistance variation vs. bending angle. The non linearity involves a time consuming calibration, more complexity of the conditioning electronics, more troubles for drift problems and the issue to establish the best fit algorithm. So here we propose methodologies and hints to linearise the sensor's electrical function.
\end{abstract}

Keywords-flex sensors, linearity, data glove, rehabilitation

\section{INTRODUCTION}

Every day, all around the world, millions of people request postural and/or motor rehabilitation. This can be for really many reasons, i.e. for traumatic or connective or degenerative musculoskeletal disorders, after traumatic events such as strokes, severe cardiac disease, prolonged rest in bed, functional losses after treatment, etc. The rehabilitation process, also known as Tertiary Prevention, intends to be a sort of therapy to restore functionality and self-sufficiency of the patient. The rehabilitation process regards not only millions of patients daily, but involves also a huge number of professionals in medical staffs, i.e. specialists, nurses, physiotherapists and therapists, social workers, psychologists, physiatrists. In addition to the patients and to the medical staffs, also many facilities are involved, since the care are given in hospitals, clinics, geriatric facilities and territorial home care.

So, for the incredible number of patients, medical staffs and facilities necessary to support the appropriate postural and motor training, the monetary costs of rehabilitation is so huge to be even really difficult to estimate.

For this reason, every effort towards a simplification of the rehabilitation route is really desirable and welcome. To this aim, our best efforts must have the purposes to reduce the cost, time, employees and facilities but possibly, at the same time, increase the effectiveness of the rehabilitation. We can, at least partially, obtain some of those requirements adopting different strategies and technologies, among which the "sensorized garments" (also known as "wearable sensors") can play a winning rule. Let's analyze the reasons.

The present day's best practice consists of a preliminary visit of the motor injured patient at a hospital division. A physiotherapist measures the residual motor functions of the patient by means of reference goniometers or other manual stuff (for instance a reflex hammer for the FUGL-MEYER assessment of physical performance for patients with stroke). On the basis of these spot measured results, the medical staff assigns to the patient a rehabilitation procedure over a period of time. But the patient needs to return periodically at the hospital division to let the physiotherapists re-perform all the procedures to measure again the residual motor function of the patient to verify any improvements. These repeated measures claim efforts and time both of the professionals and the patient, and are in any case performed with overcome methodologies.

In this scenario the sensorized garments are a valid able resource to drastically reduce those time and efforts. In fact the measurements are performed not subjected to any personnel and directly in the home environment of the patient during his/her normal daily life activities and without limitations as in a hospital scenario can be. The patient can visualize the movements he/she has to do observing a specimen avatar on a computer screen, so having non-intimidating and unambiguous suggestions. The patient can replay those movements as in a play rule, so being more motivated [1] according to the Kemp model [2]. He/she can be supported by a pc-based software which records all his/her movements and classifies them so allowing a self-evaluation of the rehabilitation progresses. An eventual supervision of qualified personnel can be remotely furnished since all recorded data can be sent via web.

In addition to all the previous considerations, the mapping of the body kinematics and the all measured values over a period of time (hours or even days) can be of strategic importance, not only for rehabilitation purposes, but even for evaluating the emotional reactions a patient can present to several events, and for realizing more ergonomic stuff necessary for the rehabilitation itself.

Within this frame, same research groups and commercial companies have developed sensorized garments for all the parts of the body, over the past 10-15 years, obtaining interesting results $[3,4,5]$. But since the hand is our first shield as a protection to fall and other kinds of mishaps, it is one of the 
main part of the body to be potentially damaged by accidents. So the motor rehabilitation highly involves the human hand. For this aspect among the sensorized garments a fundamental rule is played by the so called "data glove", which is a support glove provided with sensors to measure the flex-extension and abdu-adduction movements of the fingers and the spatial motion of the wrist.

\section{HiteG GLOVE}

The postures of the hand can be measured adopting sensors capable to transduce the movements of the finger into electrical signals. This can be obtained with sensors based on the Hall effect [6, 7], fiber optics [8], inductcoders [9], MEMS [10], optical (infrared receivers/transmitters based) systems [11], capacitive sensors [12], accelerometers [13], gyroscopes [14], piezo-resistive sensors [15]. But among all, a remarkable mention is for the bend sensor potentiometers [16] which are capable of interesting performances, in terms of measurement accuracy (stability of sensor signal after bending) and reliability, with respect to their low cost and easy applicability. In particular our research group investigated some of those bend sensors which are commercially available [17] and, on the basis of the Flexpoint bend sensor potentiometer (Fig. 1) [18], developed the Hiteh-glove (Fig. 2) so termed stands our acronym (Health Involved Technical Engineering Group).

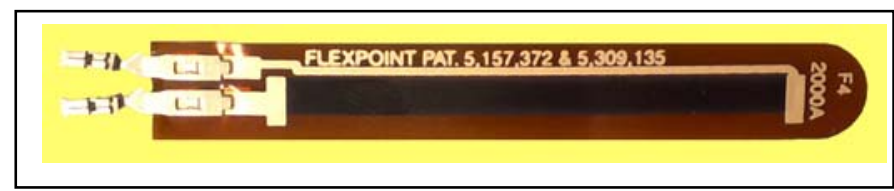

Figure 1. A Flexpoint bend sensor potentiometer. Two electric terminals visible at the left side of the figure

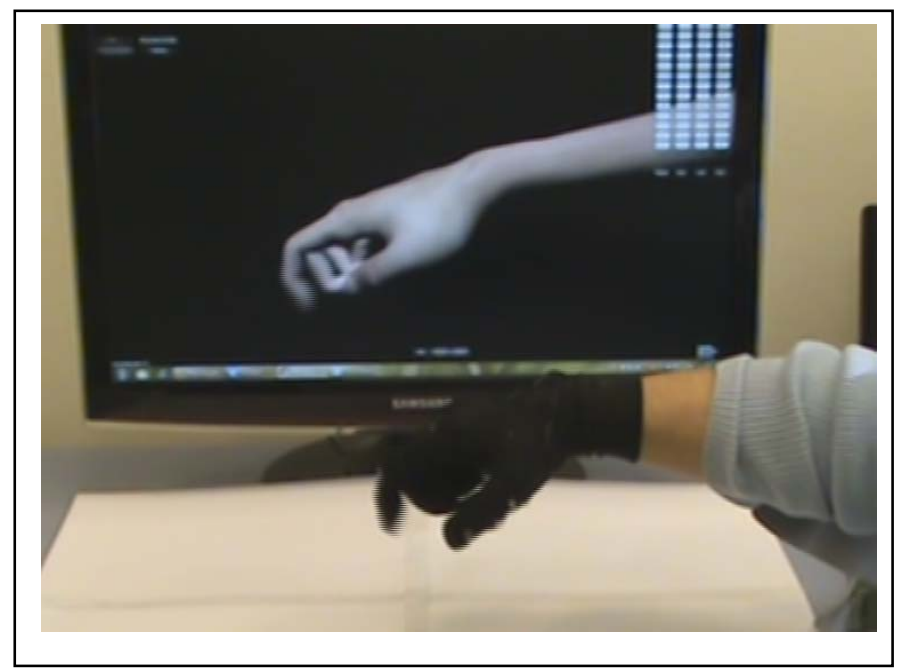

Figure 2. Hiteg glove and its avatar representation

\section{MEASURES}

We performed several measurements on these sensors with a home-made mechanical set-up. It consists of a hinge made of a knuckle through which a central circular pin is passed, and two notched leafs extend laterally from the knuckle. One of the leaf is fixed with the pin, so capable to revolve together it, while the other one is maintained fixed. A stepper motor, with its central axis jointed with the pin, can rotate the revolving leaf, so simulating the movements of a human joint [17]. The sensors were mechanically deflected 10 times each, around a pivot with $0.2 \mathrm{~cm}$ of radius, assuming angles starting from 0 till 90 degrees. Resistive values of the sensors were acquired every 10 degrees of bending for both outward and inward bending cycle. Figure 3 reports the mean values of the collected data, and represents the low standard deviation for each points, which stresses the repeatability of the measure.

Several tests were performed on two inches long Flexpoint bend sensors of three types: non encapsulated and encapsulated with polyester or polyimide materials.

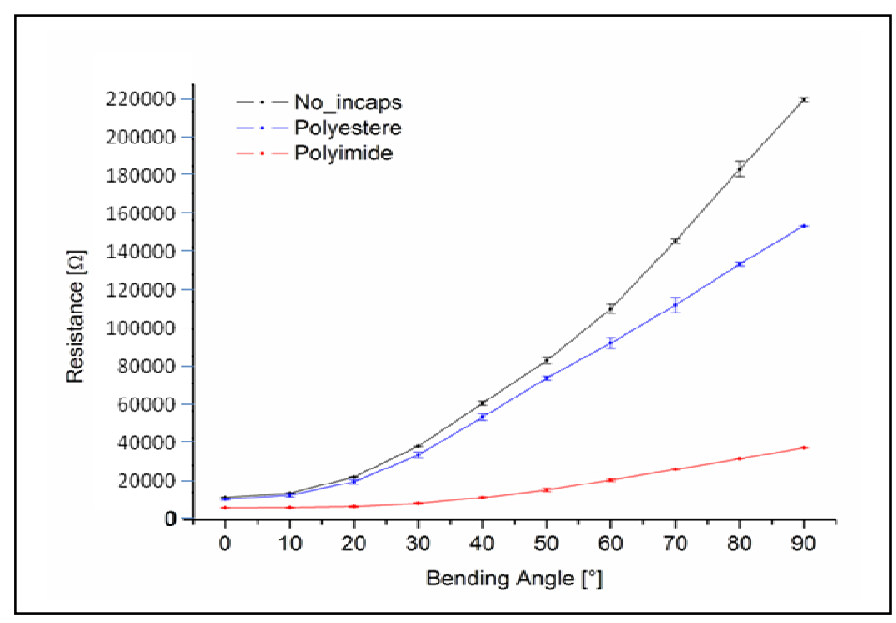

Figure 3. Resistance variation vs bending angle for three different Flexpoint sensors

Despite the interesting results of a reasonable repeatability of measure, a drawback of these sensors can be considered their non-linear relationship between measured resistance vs. angle of bending. This non linearity implies some disadvantages with respect to a linear behavior: a time consuming calibration for each individual sensor; Added complexity in designing conditioning electronics; Increased difficulty in taking into account drift problems; Difficulty to establish the best fit algorithm. Just a note: we demonstrated that this non linear behavior does not depend on the mechanical features of the sensor [19] but only on the electrical ones and we proposed an electrical model to predict the electrical behavior under different circumstances [17].

Our aim is here to point out which solutions are more effective to linearise the resistance values versus bend angle relationship of bend sensors or, simply, to overcome the problem adopting a tricky solution.

\section{METHODS}

In the year 2009, Gentner and Classen proposed to modify the two inch Flexpoint bend sensors, fixing on the sensible resistive layer a $0.2 \mathrm{~mm}$ thin unplasticised polyvinyl chloride (PVC) foil. In addiction a resistance of proper value was placed in parallel to each sensor [20]. After these modifications, the 
linear regression analysis provided changes in the coefficient of determination, $\mathrm{R}^{2}$, towards $0.9855 \div 0.9980$ values, so denoting a change of the sensor electrical characteristics in the direction of a sort of linearity. That was an interesting solutions, but a bit time consuming to realize and based on a method empirically obtained.

Here we propose an alternative novel method. It is based on the fact that our system of placing the sensors on the finger joint is different from the way find in literature. In fact the sensors are commonly inserted in a closed sleeve on top of a Lycra glove in correspondence of each finger joints [21]. Differing from that we adopted each sensor in a open pocket a bit wider but a bit shorter than the sensor itself (see Fig. 4).

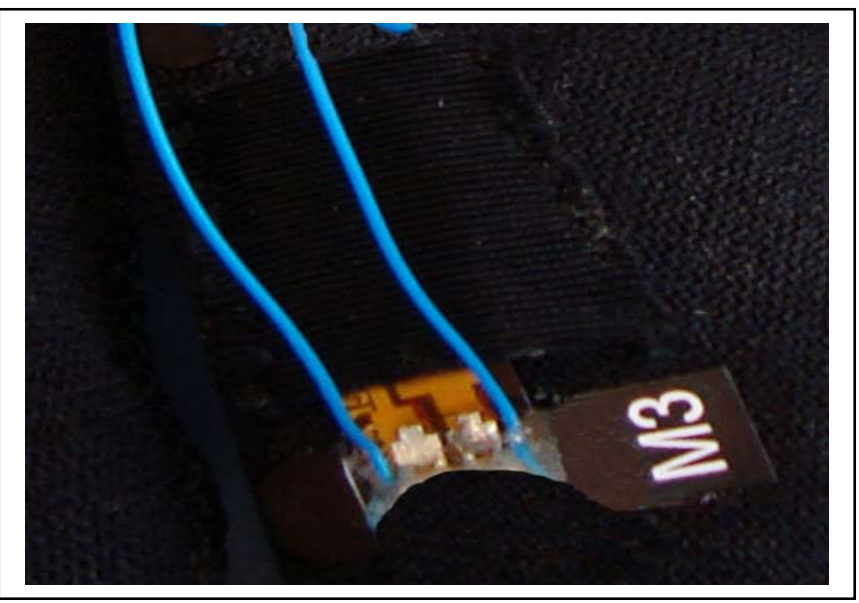

Figure 4. One-side open pocket for the sensor over one finger joint

The pocket's open end allows free sliding movements for the sensor. Only the sensor tip with the two electric terminals lodged is stitched with the pocket. All the system is then housed sewn on the Lycra glove in correspondence to a finger joint. With joint bending, this configuration does not bent always the same part of the sensor, because of a translation of the sensor itself, due to skin and glove elongation with flexion of the finger (see Fig. 5).

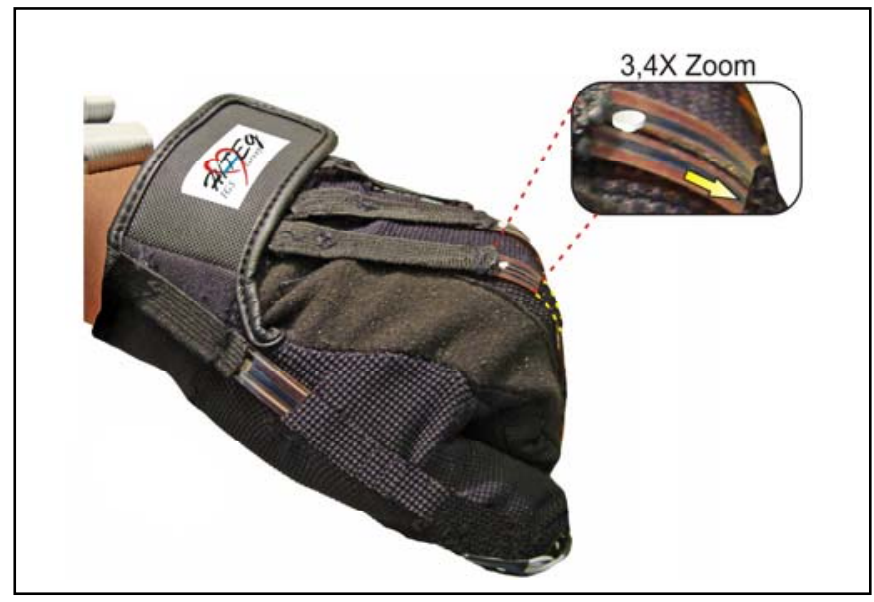

Figure 5. The sensor does not bend in the same poit, since it translates durign finger flexion
Because of that, we know exactly which part of the sensor is bent in correspondence to each angle of bending. In particular it is the part of the sensor nearest to the two electric terminals to be bent for lowest angle values and this part is the responsible for the lowest variation in resistance, as depicted in Fig. 3. For this reason our idea was to change the resistance value increasing it proportionally with the proximity of the sensor to its electric terminals. Since the resistance value is inversely proportional to the lateral section of the sensor (indicated by red arrows in Fig. 6), we changed the rectangular geometry of the sensor cutting some part of it so to obtain a triangular shape, as depicted in Fig. 6.

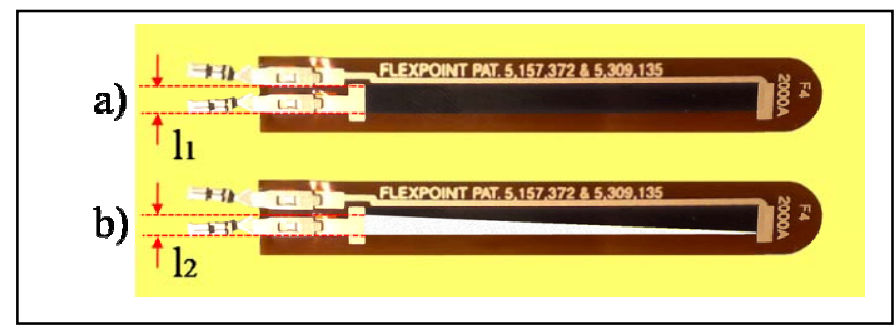

Figure 6. a) The uncutted sensor and b) the cutted triangular shaped sensor

After this modification of the geometry of the sensor (the shape of its sensible part, i.e. the black rectangular area of Fig. 6 ), from a rectangular to a triangular contour, all the previous reported measures were repeated adopting exactly the same procedures with the same set-up. The new results are reported in Fig. 7.

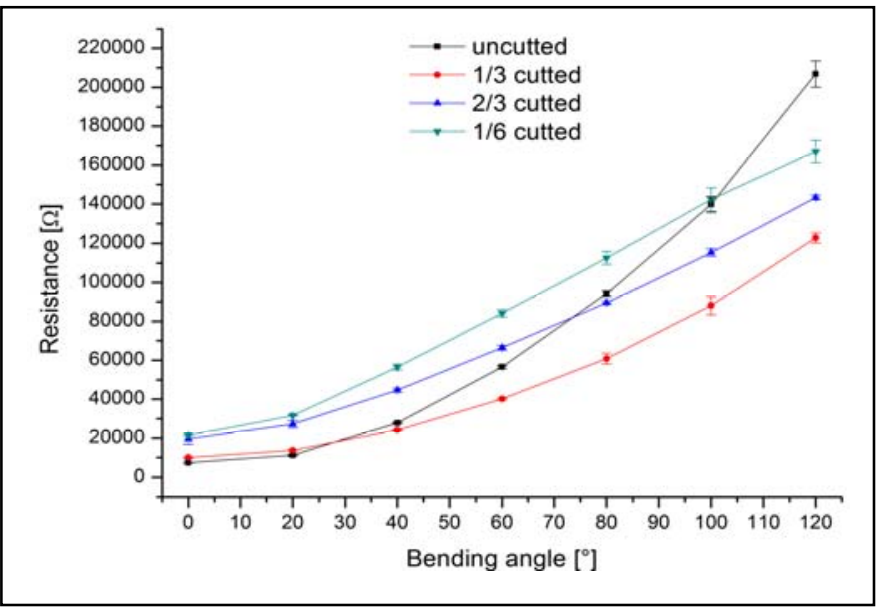

Figure 7. Linearization of the function after sensor's cuts

As it is possible to notice, we obtained an interesting modification of the function's trend towards linearization, and the linearization effect is more evident for highest amount of cuts. In fact we adopted three different amount of cuts, differing them from the " 12 " value (see Fig. 6) with respect to the " 11 " one. In particular we intend as $12 / 11=1$ for uncut sensor, $12 / 11=1 / 3$ for sensor with the lateral section reduced of $1 / 3,12 / 11=2 / 3$ and $12 / 11=1 / 6$ for sensor with the lateral section reduced of $2 / 3$ and $1 / 6$ respectively. As it is evident a new behavior of the sensor characteristic is obtained. The linear regression analysis demonstrated $\mathrm{R}^{2}=0.9893$ for $12 / 11=1 / 6$, $\mathrm{R}^{2}=0.966$ for $12 / 11=2 / 3$ and $\mathrm{R}^{2}=0.9298$ for $12 / 11=1 / 3$. So the 
amount of cut of $1 / 6$ furnishes analogous results as the previously mentioned obtained with more complex procedures of fixing the unplasticised PVC foil and placing the resistance in parallel to the sensor.

But we can face up to the linearization problem from another completely different point of view too. Paying attention to the Fig. 3, it can be noticed as the non linearity regards mostly the $0-30$ degree interval. Outer of that, the sensor characteristic can be considered almost linear. So, if we want to base our sensor exploitation within a linear electrical characteristic, it is sufficient to utilize the sensor limiting its bending excursion outer from that interval, for angles greater than 30 degrees. Limiting the sensor bending excursion within the 30-90 degree range, the linear regression analysis presents $\mathrm{R}^{2}=0.9789$.

This possible usage of the sensors can be realized with a sort of "bridge" geometrical configuration of application of the sensors, as showed in Figure 8. In this way the sensors are already bent with a 30 degree angle when the hand is completely opened, and increase more and more their bending angle values closing the hand till a fist position.

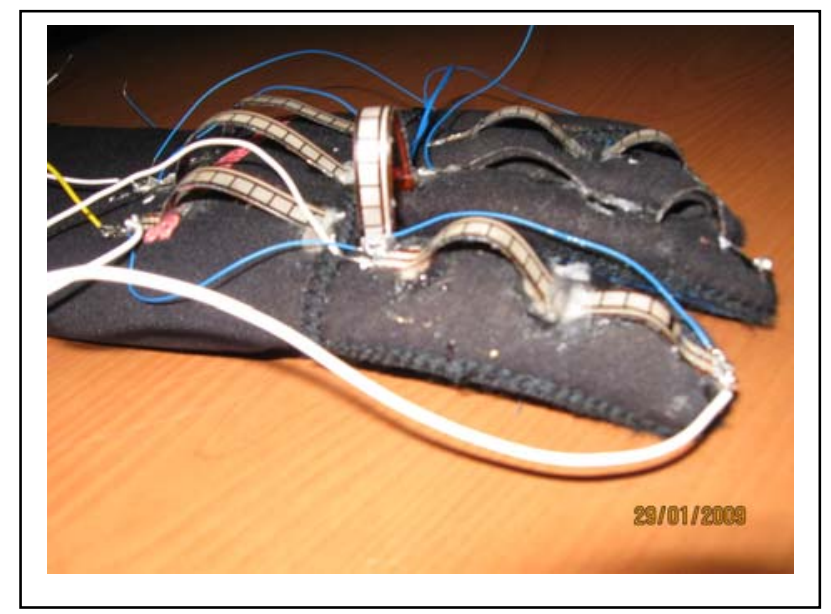

Figure 8. "Bridge" geometric configuration of application of the sensors

\section{CONCLUSIONS}

The flex sensors can be a cheap and helpful tool to realize data gloves with good performances in terms of reliability and stability of sensor signal after bending. Although these remarkable features, the resistance variation vs. bending angle function is not linear, so some drawbacks must be taken into account. Here we offer a survey on methodologies and propose hints to linearise that function, validating the results with proper measures.

\section{ACKNOWLEDGMENT (HEADING 5)}

I would like to thank all the Hiteg group of the Department of Electronic Engineering of my University.

\section{REFERENCES}

[1] N. Maclean et al. "The Concept of Patient Motivation. A Qualitative Analysis of Stroke Professionals' Attitudes. Stroke 2000, 33, 444-448

[2] Bryan J. Kemp "Motivation, Rehabilitation and aging: A conceptual model. Topics in Geriatric Rehabilitation", 1988, Vol.3, Issue 3, 41-51

[3] T. Giorgino, P. Tormene, G. Maggioni, D. Capozzi, S. Quaglini, C. Pistarini, "Assessment of sensorized garments as a flexible support to self-administered post-stroke physical rehabilitation" Eur J Phys Rehabil Med 2009;45:75-84

[4] F. Lorussi, A. Tognetti, M. Tescioni, G. Zupone, R. Bartalesi,. D. De Rossi "Electroactive Fabrics for Distributed, Confortable and Interactive Systems" in Techn. and Informatics vol.117, Personalized Health Management Systems, Ed. Chris D. Nugent et al., IOS Press, 2005

[5] E. R. Post, M. Orth, P. R. Russo, N. Gershenfeld "E-broidery: design and fabrication of textile-based computing", IBM Systems Journal Volume 39 Issue 3-4, July 2000

[6] O. Portillo-Rodriguez, CA Avizzano, E. Sotgiu, S. Pabon, A. Frisoli, J. Ortiz, M. Bergamasco "A wireless Bluetooth Dataglove based on a novel goniometric sensors." 16th IEEE International Symposium on Robot \& Human Interactive Communication. Jeju Island, Korea. August 26 - 29, 2007. ISBN/ISSN: 978-1-4244-1635-6.

[7] L. Dipietro, A. M. Sabatini, and P. Dario, "Evaluation of an instrumented glove for hand-movement acquisition," J. Rehabil. Res. Dev., vol. 40, no. 2, pp. 179-190, Mar./Apr. 2003.

[8] S. Wise, W. Gardner, E. Sabelman, E. Valainis, Y. Wong, K. Glass, J. Drace, J. M. Rosen, "Evaluation of a fiber optic glove for se ${ }^{\circ}$ automated goniometric measurements" Journal of Rehabilitation Research and Development Vol . 27 No. 4, 1990, Pages 411-424

[9] T Kuroda, Y Tabata, A Goto, H Ikuta, M Murakami "Consumer price data-glove for sign language recognition" Proc. 5th Intl Conf. Disability, Virtual Reality \& Assoc. Tech., Oxford, UK, 2004; ISBN 0704911442

[10] J. K. Perng, B. Fisher, S. Hollar, and K. S. J. Pister, "Acceleration sensing glove (ASG)," in Proc. IEEE Int. Symp. Wearable Comput., 1999, pp. 178-180.

[11] C.-S. Fahn , H. Sun "Development of a Sensory Data Glove Using Neural-Network-Based Calibration" Proc. ICAT 2000, The Tenth International Conference on Artificial Reality and Tele-existence, October 25-27, 2000, National Taiwan University, Taipei, Taiwan

[12] N. Karlsson, B. Karlsson, and P. Wide, "A glove equipped with finger flexion sensors as a command generator used in fuzzy control system," IEEE Trans. Instrum. Meas., vol. 47, no. 5, pp. 1330-1334, Oct. 1998.

[13] J. L. Hernandez-Rebollar, N. Kyriakopoulos, and R.W. Lindeman, "The AcceleGlove: A whole-hand input device for virtual reality," in Proc. SIGGRAPH, 2002, p. 259.

[14] B. S. Soh, Y. S. Kim, and S. G. Lee, "Improving the usability of a wearable input device SCURRY," presented at the Int. Conf. Ubiquitous Comput., Nottingham, U.K., 2004.

[15] D. L. Gardner, “The Power Glove,” Des. News, 1898, vol.45, pp. 63-68

[16] N. W. Williams, J. M. T. Penrose, C. M. Caddy, E. Barnes, D. R. Hose, P. Harley "A goniometric glove for clinical hand assessment" Journal of Hand Surgery (British and European Volume, 2000) 25B: 2: 200-207

[17] G. Orengo, G. Saggio, S. Bocchetti, F. Giannini "Advanced characterization of piezoresistive sensors for human body movement tracking" Nano-bio circuits fabrics and systems, ISCAS 2010, May 30th - June 2nd, Paris (France), pp.1181-1184

[18] Online http://www.flexpoint.com/

[19] G. Saggio, P. Bisegna, G. Latessa, S. Bocchetti "Mechanical modeling of bend sensors exploited to measure human joint movements" 1th IEEE International WoWMoM Workshop on Interdisciplinary Research on EHealth Services and Systems, IREHSS 2009, Kos (Greece) June 2009

[20] R. Gentnera, J. Classena "Development and evaluation of a low-cost sensor glove for assessment of human finger movements in neurophysiological settings" Journal of Neuroscience Methods 178 (2009) 138-147

[21] LK. Simone, N. Sundarrajan, X. Luo, Y. Jia, D.G. Kamper “A low cost instrumented glove for extended monitoring and functional hand assessment" J Neurosci Methods 2007;160:335-348 